EPJ Web of Conferences 97,00007 (2015)

DOI: 10.1051/epjconf/ 20159700007

(C) Owned by the authors, published by EDP Sciences, 2015

\title{
Resonance Production with the LHCb Experiment
}

\author{
N. H. Brook ${ }^{1, a}$ \\ on behalf of the LHCb collaboration. \\ ${ }^{1}$ UCL, Department of Physics and Astronomy, Gower Street, London WC1E 6BT, United Kingdom
}

\begin{abstract}
Studies of strange and charm resonance production in the forward region provide important input to the importance to the understanding of QCD models. The latest studies at $\mathrm{LHCb}$ of resonance production in both proton-proton and proton-lead collisions are presented, with particular emphasis on charmonium production including the production of $Z(4430)$.
\end{abstract}

\section{Introduction}

Charmonium is an ideal system in which to study Quantum Chromodynamics. Although the $J / \psi$ meson was identified over 40 years ago in many ways it still proves to be an enigma. Theoretically the production of charmonia is factorised, with the production of a $c-\bar{c}$ pair in perturbative short-distance processes and with the creation of the charmonium described by long-distance, non-perturbative processes. The framework often used for these calculations is known as NRQCD [1]. Within this framework the perturbative part can be described from contributions from the colour singlet (CS) and the colour octet $(\mathrm{CO})$ matrix elements. The theoretical models struggle to describe the data from the Tevatron consistently between the $p_{T}$ differential cross section and the polarisation measurement of the $J / \psi$.

QCD-motivated models for hadrons predict an assortment of "exotic" hadrons that have structures that are more complex than the traditional quark-parton model [2]. The observation of charmoniumlike states has generated much interest in exotic meson spectroscopy. The $X(3872)$ particle was discovered in 2003 by the Belle collaboration [3] in the $B^{ \pm} \rightarrow X(3872) K^{ \pm}$with $X(3872) \rightarrow \psi \pi^{+} \pi^{-}$, later confirmed by other collaborations [4]. The proximity of the $X(3872)$ mass to the $\bar{D}^{* 0} D^{0}$ threshold is suggestive that the $X(3872)$ is a loosely bound $\bar{D}^{* 0} D^{0}$ "molecule". A further, more exotic, possibility is that the $X(3872)$ is a tetraquark state. The Belle collaboration have also found evidence for a narrow $Z(4430)^{-}$peak in the $\psi(2 S) \pi$ mass distribution. As the minimal quark content of such a state is $c \bar{c} d \bar{u}$, this observation could also be interpreted as evidence for the existence of another meson beyond the quark parton model.

\section{$2 \mathrm{LHCb}$ Detector}

The LHCb detector [6] is a single-arm forward spectrometer covering the pseudorapidity range $2<\eta<5$. The detector includes a high-precision tracking system consisting of a silicon-strip vertex

\footnotetext{
ae-mail: n.brook@ucl.ac.uk
}

This is an Open Access article distributed under the terms of the Creative Commons Attribution License 4.0, which permits unrestricted use, distribution, and reproduction in any medium, provided the original work is properly cited. 


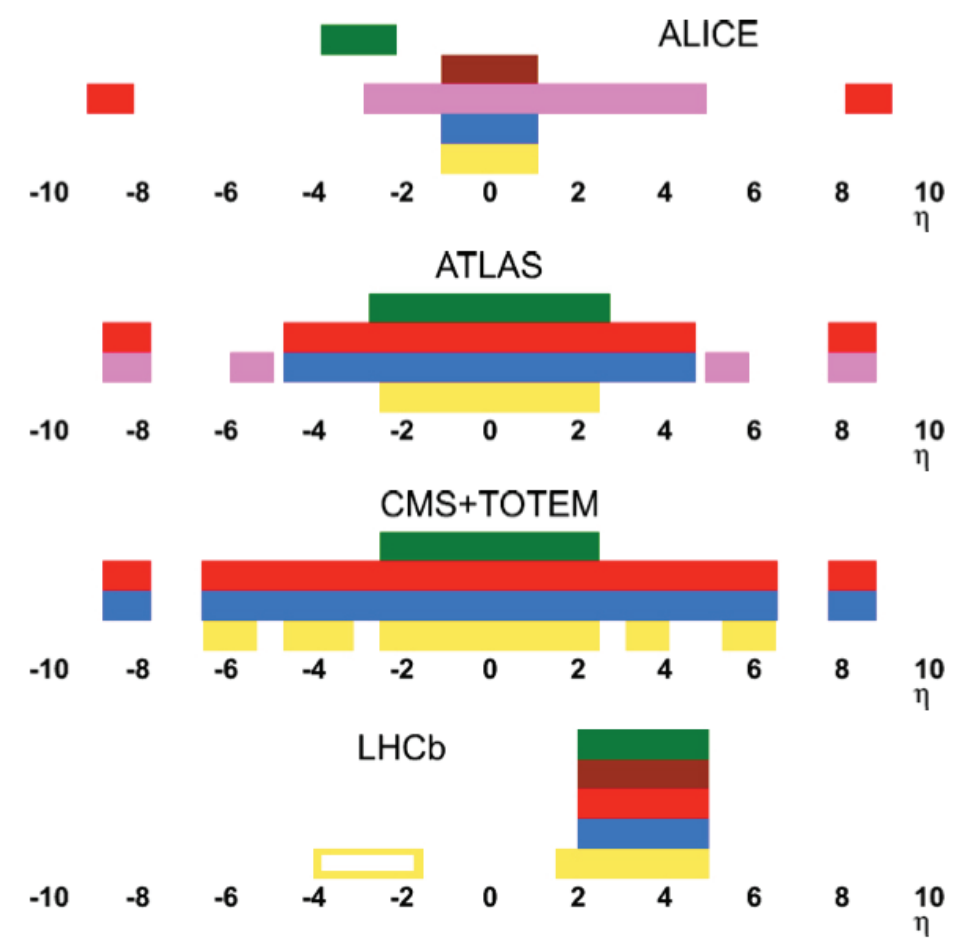

Figure 1: A generic diagram illustrating the acceptance of the LHC experiments. The unfilled box indicates only partial coverage.

detector surrounding the $p p$ interaction region, a large-area silicon-strip detector located upstream of a dipole magnet with a bending power of about $4 \mathrm{Tm}$, and three stations of silicon-strip detectors and straw drift tubes placed downstream. The combined tracking system provides a momentum measurement with relative uncertainty that varies from $0.4 \%$ at $5 \mathrm{GeV} / \mathrm{c}$ to $0.6 \%$ at $100 \mathrm{GeV} / \mathrm{c}$, and impact parameter resolution of $20 \mu \mathrm{m}$ for tracks with high transverse momentum. Charged hadrons are identified using two ring-imaging Cherenkov detectors. The calorimeter system consists of a scintillating pad detector (SPD) and a pre-shower system (PS), followed by electromagnetic (ECAL) and hadron calorimeters. The SPD and PS are designed to distinguish between signals from photons and electrons. Muons are identified by a system composed of alternating layers of iron and multiwire proportional chambers. The acceptance of the LHCb spectrometer is complementary to that of the other LHC detectors as can be seen in figure 1. The trigger [7] consists of a hardware stage, based on information from the calorimeter and muon systems, followed by a software stage where a full event reconstruction is applied.

The $\mathrm{LHCb}$ detector also took data during the $\mathrm{LHC} p \mathrm{~Pb}$ run in early 2013, corresponding to an integrated luminosity of $1.1 \mathrm{nb}^{-1}\left(0.5 \mathrm{nb}^{-1}\right)$ for forward (backward) collisions. The instantaneous luminosity was around $5 \times 10^{27} \mathrm{~cm}^{-2} \mathrm{~s}^{-1}$, five orders of magnitude below the typical LHCb luminosity for pp collisions. The energy of the proton beam was $4 \mathrm{TeV}$, while that of the lead beam was $1.58 \mathrm{TeV}$ per nucleon, resulting in a centre-of-mass energy of the nucleon-nucleon system of $5.02 \mathrm{TeV}$. Since the energy per nucleon in the proton beam is significantly larger than that in the lead beam, the 
nucleon-nucleon centre-of-mass system has a rapidity in the laboratory frame of $+0.465(-0.465)$ for $p \mathrm{~Pb}$ forward (backward) collisions, where the rapidity is defined with respect to the proton beam. This results in a shift of the rapidity coverage in the nucleon-nucleon centre-of-mass system, ranging from about 1.5 to 4.0 for forward $p \mathrm{~Pb}$ collisions and from -5.0 to -2.5 for backward $p \mathrm{~Pb}$ collisions. The hardware trigger during this period was simply an interaction trigger, which rejects empty events. The software trigger requires one well-reconstructed track with hits in the muon system and a $p_{T}$ greater than $600 \mathrm{MeV} / \mathrm{c}$.

\section{Results}

\subsection{Charmonium polarisation in $p p$ collisions}

The three polarization states of charmonium can be specified in terms of a chosen coordinate system in the rest frame of the meson. Several polarization frames can be used for the analysis. In the helicity frame [8] the polarization axis coincides with the flight direction of the charmonium in the centre-of-mass frame of the colliding hadrons. The angular decay distribution is described by

$$
\frac{d^{2} N}{d \cos \theta d \phi}=1+\lambda_{\theta} \cos ^{2} \theta+\lambda_{\theta \phi} \sin 2 \theta \cos \phi+\lambda_{\phi} \sin ^{2} \theta \cos 2 \phi
$$

where $\theta$ is the polar angle between the direction of the positive lepton and the chosen polarization axis, and $\phi$ is the azimuthal angle, measured with respect to the production plane.

The fit results for the three parameters $\lambda_{\theta}, \lambda_{\theta \phi}$ and $\lambda_{\phi}$ for $J / \psi$ are reported in [9]. The parameter $\lambda_{\theta}$ is also shown in figure $2 \mathrm{a}$ as a function of the $p_{T}$ of the $J / \psi$ meson, for different rapidity (y) bins. The polarization parameters $\lambda_{\phi}$ and $\lambda_{\theta \phi}$ in the helicity frame are consistent with zero within the uncertainties. The measured value of $\lambda_{\theta}$ shows a small longitudinal polarization. A similar analysis was performed for the $\psi(2 S)$ meson [10]. Again the parameter $\lambda_{\theta}$ is shown in figure $2 \mathrm{~b}$ as a function of transverse momentum, $p_{T}$, for different bins of $y$. The polarisation parameter $\lambda_{\theta}$ is consistent with zero, with no significant dependence on $p_{T}$ or $y$ of the $\psi(2 S)$ meson. The polarisation parameters $\lambda_{\theta \phi}$ and $\lambda_{\phi}$ are also consistent with zero. However the frame-invariant polarisation parameter $\lambda_{\text {inv }}=\frac{\lambda_{\theta}+3 \lambda_{\phi}}{1-\lambda_{\phi}}$ is consistent with a negative polarisation with no strong dependence on $p_{T}$ and $y$. Overall the coloursinglet model (CSM) [11] is disfavoured by both sets of data and the NLO NRQCD from [12] gives the best description of the data but overshoots the $\psi(2 S)$ data at $p_{T}>10 \mathrm{GeV} / \mathrm{c}$.

\subsection{Heavy Ion Charmonium Production}

The numbers of prompt $J / \psi$ mesons and $J / \psi$ from $b$-production have been studied at LHCb in $p \mathrm{~Pb}$ collisions [14] in bins of the kinematic variables $y$ and $p_{T}$. The relevant production mechanisms are determined by performing combined extended maximum likelihood fits to the unbinned distributions of dimuon mass and the pseudoproper time in each kinematic bin. The pseudoproper time of the $J / \psi$ meson is defined as

$$
t_{z}=\frac{\left(z_{J / \psi}-z_{\mathrm{PV}}\right) \times M_{J / \psi}}{p_{z}},
$$

where $z_{J / \psi}, z_{\mathrm{PV}}$ are the $z$ positions of the $J / \psi$ decay vertex and the primary vertex respectively, $p_{z}$ is the $z$-component of the measured $J / \psi$ momentum and $M_{J / \psi}$ is the known $J / \psi$ mass.

The single differential production cross-sections as functions of $p_{T}$ and $y$, for both samples in the $p \mathrm{~Pb}$ forward region, are shown in figure 3. The integrated production cross sections for prompt $J / \psi$ mesons and $J / \psi$ from $b$ with $p_{T}<14 \mathrm{GeV} / \mathrm{c}$ in the forward and backward regions respectively are: 


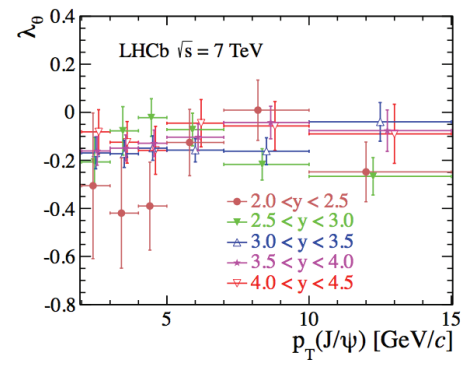

(a) Measurements of $J / \psi$ polarisation [9].

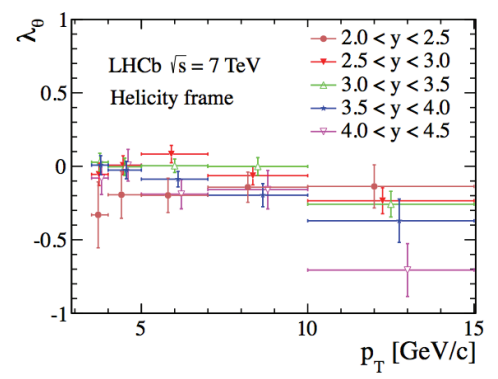

(b) Measurements of $\psi(2 S)$ polarisation [10].

Figure 2: Measurements of $\lambda_{\theta}$ in bins of $p_{T}$ for five rapidity bins in the helicity frame. The error bars represent the statistical and systematic uncertainties added in quadrature.

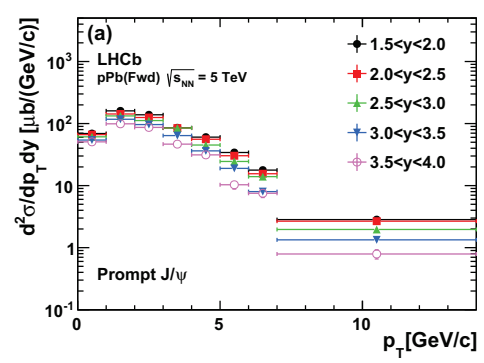

(a) Prompt $J / \psi$ meson production.

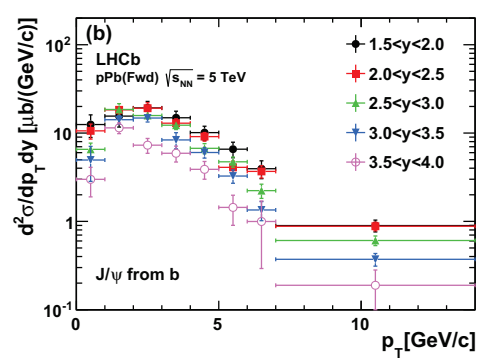

(b) $J / \psi$ meson production from b's.

Figure 3: Double differential production cross sections for $J / \psi$ meson production for forward collisions [14].

$$
\begin{aligned}
\sigma_{p A}(\text { prompt }) & =1168 \pm 15 \pm 54 \mu b \\
\sigma_{p A}(\text { from } b) & =166.0 \pm 4.1 \pm 8.2 \mu b \\
\sigma_{A p}(\text { prompt }) & =1293 \pm 42 \pm 75 \mu b \\
\sigma_{A p}(\text { from } b) & =118.2 \pm 6.8 \pm 11.7 \mu b
\end{aligned}
$$

The reference production cross-section in $p p$ collisions at $5 \mathrm{TeV}$ for prompt $J / \psi$ mesons is $4.79 \pm$ $0.22 \pm 0.15 \mu \mathrm{b}$, and that for $J / \psi$ from $b$ is $0.47 \pm 0.04 \pm 0.01 \mu \mathrm{b}$ [13]

Nuclear effects are usually characterized by the nuclear modification factor, defined as the production cross-section of a given particle in $p A$ collisions divided by that in $p p$ collisions and the number of colliding nucleons in the nucleus (given by the atomic number $A$ ),

$$
R_{p A}(y, \sqrt{s})=\frac{1}{A} \frac{\frac{d \sigma_{p A}(y, \sqrt{s})}{d y}}{\frac{d \sigma_{p p}(y, \sqrt{s})}{d y}} .
$$




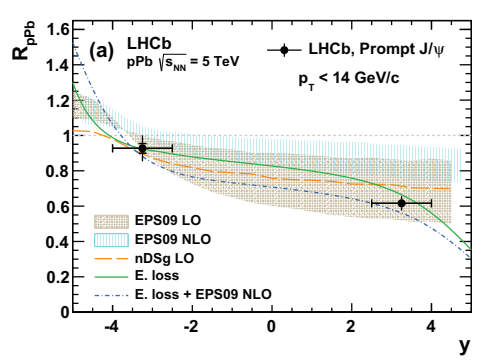

(a) Prompt $J / \psi$ meson production.

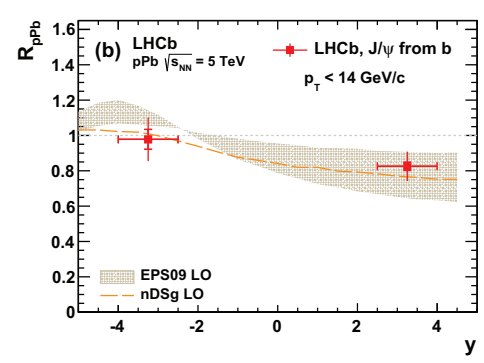

(b) $J / \psi$ meson production from b's.

Figure 4: Nuclear modification factor $R_{p \mathrm{~Pb}}$ as a function of $y$ [14] together with theoretical predictions.

Figure 4 a shows the nuclear modification factor for prompt $J / \psi$ production, together with several theoretical predictions [15]. A suppression of about $40 \%$ at large rapidity is observed for prompt $J / \psi$ production. The measurements agree with most predictions. However, the calculation based on NLO colour evaporation model with the EPS09 shadowing parameterisation provides a less good description of the measurement in the forward region, though there is a better description when coherent energy loss is included. Figure $4 \mathrm{~b}$ shows the nuclear modification factor for $J / \psi$ from $b$, together with the theoretical predictions [16]. The data show a modest suppression of $J / \psi$ from $b$-production in $p \mathrm{~Pb}$ forward region, with respect to that in $p p$ collisions. This is the first indication of the suppression of $b$-hadron production in $p \mathrm{~Pb}$ collisions. The theoretical predictions agree with the measurement in the forward region. In the backward region the agreement is not as good. The observed production suppression of $J / \psi$ from $b$ with respect to $p p$ collisions is smaller than that of prompt $J / \psi$, which is consistent with theoretical predictions.

\subsection{Exotic Mesons}

Measurements of $X(3872)$ production at hadron colliders, where most of the production is prompt rather than from $B$-hadron decays, may shed light on the nature of this particle. In particular, whether a molecular nature of the $X(3872)$ is consistent with results from the Tevatron [17]. The observed $\mathrm{X}(3872)$ signal at $\mathrm{LHCb}[18]$ is used to measure the product of the inclusive production cross-section $\sigma(p p \rightarrow X(3872)+$ anything $)$ and the branching fraction $\mathcal{B}\left(X(3872) \rightarrow J / \psi \pi^{+} \pi^{-}\right)$

$$
\sigma(p p \rightarrow X(3872)+\text { anything }) \mathcal{B}\left(X(3872) \rightarrow J / \psi \pi^{+} \pi^{-}\right)=5.4 \pm 1.3(\text { stat }) \pm 0.8(\text { syst }) \mathrm{nb}
$$

in the kinematic region $2.5<y<4.5$ and $5<p_{T}<20 \mathrm{GeV} / \mathrm{c}$.

$\mathrm{LHCb}$ has undertaken a five-dimensional angular correlations of the $B^{+} \rightarrow$ $X(3872) K^{+}, X(3872) \rightarrow \pi^{+} \pi^{-} J / \psi, J / \psi \rightarrow \mu^{+} \mu^{-}$decay chain [19]. The angular correlations in the $B^{+}$ decay carry information about the $X(3872)$ quantum numbers. To discriminate between the $1^{++}$and $2^{-+}$assignments LHCb uses the likelihood-ratio test. The probability density function for each $J^{P C}$ hypothesis, $J_{X}$, is defined in the $5 \mathrm{D}$ angular space $\Omega=\left(\cos \theta_{X}, \cos \theta_{\pi \pi}, \Delta \phi_{X, \pi \pi}, \cos \theta_{J / \psi}, \Delta \phi_{X, J / \psi}\right)$ by the normalized product of the expected decay matrix element $(\mathcal{M})$ squared and of the reconstruction efficiency, where $\theta_{X}, \theta_{J / \psi}$ and $\theta_{\pi \pi}$ are the $X, J / \psi$ and $\pi \pi$ helicity angles, $\Delta \phi_{X, \pi \pi}$ and $\Delta \phi_{X, J \psi}$ are the differences between the decay planes. The $C$-parity is already known to be positive as the decay 


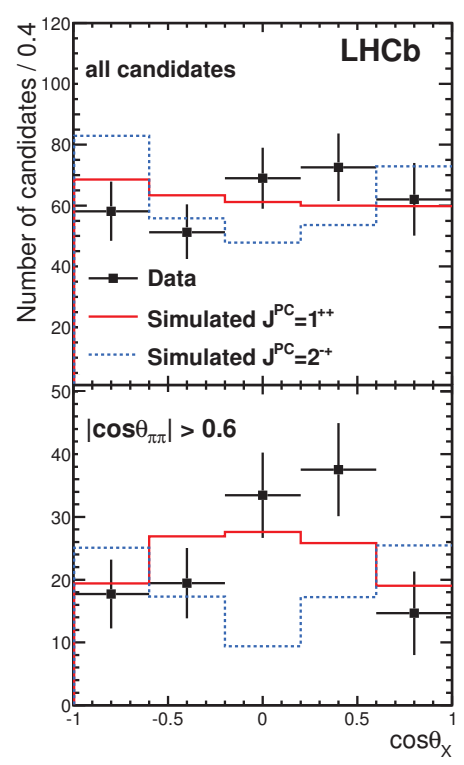

Figure 5: Background-subtracted distribution of $\cos \theta_{X}$ for (top) all candidates and for (bottom) candidates with $\left|\cos \theta_{\pi \pi}\right|>0.6$ for the LHCb data [18] compared to the expected distributions for the $J^{P C}=1^{++}$(red solid histogram) and $J^{P C}=2^{-+}$hypotheses (blue dashed histogram).

$X(3872) \rightarrow \gamma J / \psi$ has been observed. The fits correspond to a $8.2 \sigma$ rejection of the $2^{-+}$hypothesis. $\mathrm{LHCb}$ projected the data, as an additional goodness of test fit, onto five 1D and ten 2D binned distributions in all five angles and their combinations. They are all consistent with the distributions expected for the $1^{++}$hypothesis whilst there are inconsistencies with the distributions expected for the $2^{-+}$hypothesis. The most significant inconsistency is observed for the $2 \mathrm{D}$ projections onto $\cos \theta_{X}$ and $\cos \theta_{\pi \pi}$. The separation between the $1^{++}$and $2^{-+}$hypotheses increases when using correlations between these two angles, as seen in figure 5 .

Evidence for the decay mode $X(3872) \rightarrow \psi(2 S) \gamma$ in $B^{+} \rightarrow X(3872) K^{+}$decays is reported by $\mathrm{LHCb}$ [20] with a significance of $4.4 \sigma$. The ratio of the branching fraction of the $X(3872) \rightarrow \psi(2 S) \gamma$ decay to that of the $X(3872) \rightarrow J / \psi \gamma$ decay is measured to be

$$
R_{\psi \gamma}=\frac{\mathcal{B}(X(3872) \rightarrow \psi(2 S) \gamma)}{\mathcal{B}(X(3872) \rightarrow J / \psi \gamma)}=2.46 \pm 0.64 \pm 0.29
$$

where the first uncertainty is statistical and the second is systematic. The measured value agrees with expectations for a pure charmonium interpretation of the $X(3872)$ state $\left(1.2<R_{\psi \gamma}<15\right)$ and a mixture of charmonium and molecular interpretations $\left(1.2<R_{\psi \gamma}<5\right)$. However, it does not support a pure $D \bar{D}^{*}$ molecular interpretation of the $X(3872)$ state, which has no $X(3872) \rightarrow \psi(2 S) \gamma$ decay.

The existence of charged charmonium-like states has been a topical since the Belle collaboration found evidence for a narrow $Z(4430)$ peak in $B^{0} \rightarrow \psi(2 S) \pi K$ decays. LHCb has studied resonant structures in those decays and analyzed them by performing a $4 \mathrm{D}$ fit of the decay amplitude on about $25 \mathrm{k}$ candidates [21]. The background-subtracted and efficiency-corrected $m_{\psi(2 S) \pi}$ distribution is show in figure 6. Amplitude models are fitted to the data using an unbinned maximum likelihood method. The formalism and notation follows that outlined in [22] of a 4D amplitude dependent 


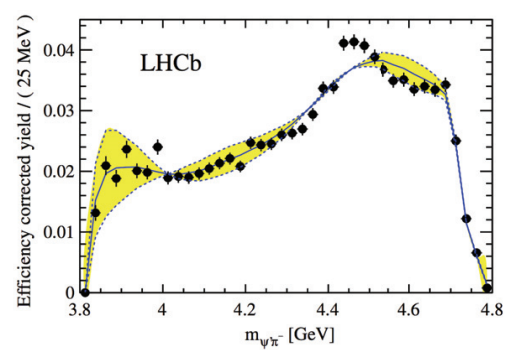

Figure 6: Background-subtracted and efficiency-corrected $m_{\psi(2 S) \pi}$ distribution (black data points), superimposed with the reflections of $\cos \theta_{K^{*}}$ moments up to order four allowing for $J\left(K^{*}\right) \leq 2$ (blue line) and their correlated statistical uncertainty (yellow band bounded by blue dashed lines) [21].

on $\Phi=\left(m_{K \pi}^{2}, m_{\psi(2 S) \pi}^{2}, \cos \theta_{\psi(2 S)}, \phi\right)$, where $\theta_{\psi(2 S)}$ is the $\psi(2 S)$ helicity angle and $\phi$ is the angle between the $K^{*}$ and $\psi(2 S)$ decay planes in the $B^{0}$ rest frame. The amplitude model includes all known $K^{* 0} \rightarrow K \pi$ resonances with nominal mass within or slightly above the kinematic limit $(1593 \mathrm{MeV})$ in $B^{0} \rightarrow \psi(2 S) K \pi$ decays. A non-resonant $J=0$ term is also included in the fits. Fits with all $K^{*}$ components and two different $J=0$ models do not give a satisfactory description of the data; the $p_{\chi^{2}}$ is below $2 \times 10^{-6}$, equivalent to a $4.8 \sigma$ discrepancy. If the $K_{3}^{*}(1780)$ component is excluded from the amplitude, the discrepancy increases to $6.3 \sigma$. This is supported by an independent study using the model-independent approach developed by the BaBar collaboration [23], which does not constrain the analysis to any combination of known $K^{*}$ resonances, but merely restricts their maximal spin, as illustrated figure 6 .

If a $Z(4430)$ component with $J^{P}=1^{+}$(known as $Z_{1}$ ) is added to the amplitude, the $p_{\chi^{2}}$ reaches $4 \%$ when all the $K^{*} \rightarrow K \pi$ resonances with a pole mass below the kinematic limit are included. The $p_{\chi^{2}}$ rises to $12 \%$ if the $K^{*}(1680)$ is also added, but fails to improve when the $K_{3}^{*}(1780)$ is additionally included. To discriminate between various $J^{P}$ assignments LHCb uses a likelihood-ratio test $\Delta(-2 \ln L)$ between the different spin hypotheses [24]. Relative to the $1^{+}$scenario LHCb rule out the $0^{-}, 1^{-}, 2^{+}$and $2^{-}$hypotheses by at least $9.7 \sigma, 15.8 \sigma, 16.1 \sigma$ and $14.6 \sigma$ respectively. The measured mass of the $Z_{1}$ is $4475 \pm 7_{-25}^{+15} \mathrm{MeV}$ with a width, $\Gamma=172 \pm 13_{-34}^{+37} \mathrm{MeV}$, and with an amplitude fraction of $f_{Z_{1}}=\left(5.9 \pm 0.9_{-3.3}^{+1.5}\right) \%$. In the amplitude fit, the $Z_{1}$ is represented by a Breit-Wigner amplitude, where the magnitude and phase vary according to an approximately circular trajectory in the Argand diagram. LHCb performed an additional fit to the data, in which the $Z_{1}$ amplitude is represented as a combination of independent complex amplitudes at six equidistant points in $m_{\psi(2 S)}^{2} \pi$. The resulting Argand diagram, shown in figure 7 , is consistent with that of a resonance.

\section{Summary}

LHCb has a very active research programme on charmonium physics in both $p p$ and $p \mathrm{~Pb}$ collisions at the LHC, including the investigation of exotic mesons.

The $J / \psi$ polarisation measurement show $\lambda_{\theta}$ with a small longitudinal polarization while the results for $\lambda_{\theta \phi}$ and $\lambda_{\phi}$ are consistent with zero. The measured values for $\psi(2 S)$ of $\lambda_{\theta \phi}$ and $\lambda_{\phi}$ are small. The $\lambda_{\theta}$ distribution in the helicity frame shows that the $\psi(2 S)$ meson exhibits neither large transverse nor longitudinal polarisation. The results, that are obtained for prompt $J / \psi$ production, including the feed-down from higher excited states, contradict the CSM predictions for direct $J / \psi$ production, both in the size of the polarization parameters and the $p_{T}$ dependence. Also the prompt $\psi(2 S)$ polarisation 


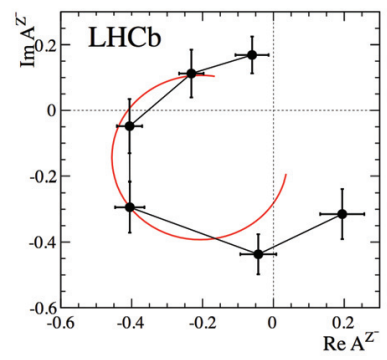

Figure 7: Fitted values of the $Z_{1}$ amplitude in six $m_{\psi(2 S)}^{2} \pi$ bins, shown in an Argand diagram. The red curve is the prediction from the Breit-Wigner formula with a resonance mass (width) of 4475 (172) $\mathrm{MeV}$ and magnitude scaled to intersect the bin with the largest magnitude centred at $(4477 \mathrm{MeV})^{2}$ [21].

measured at $\mathrm{LHCb}$ disagrees with the CSM predictions both in the size of the polarization parameters and the $p_{T}$ dependence.

The production of prompt $J / \psi$ mesons and of $J / \psi$ from $b$-hadron decays is studied in $p \mathrm{~Pb}$ collisions. The nuclear modification factor $R_{p \mathrm{~Pb}}$ indicates that cold nuclear matter effects are less pronounced for $J / \psi$ mesons from $b$-hadron decays, hence for $b$ hadrons, than for prompt $J / \psi$ mesons and shows that it is necessary to include cold nuclear matter effects in the interpretation of quark-gluon plasma signatures in heavy-ion collisions.

LHCb unambiguously established that the values of total angular momentum, parity and chargeconjugation eigenvalues of the $X(3872)$ state are $1^{++}$. The measured decay modes of the $X(3872)$ with a photon agrees with expectations for a pure charmonium interpretation of the state or a molecularcharmonium mixture interpretations but does not support a pure $D \bar{D}^{*}$ molecular interpretation. LHCb confirmed the existence of the $Z(4430)$ resonance and establishes its spin-parity to be $1^{+}$. For the first time the resonant character of $Z(4430)$ is confirmed, strongly suggesting it as a charged four-quark state.

\section{References}

[1] G.T. Bodwin, E. Braaten anf G.P. Lepage, Phys. Rev. D 51,1125 (1995).

[2] M. Gell-Mann, Phys. Lett. 8. 214 (1964).

[3] BELLE collaboration, S.-K. Choi et al., Phys. Rev. Lett. 91, 262001 (2003).

[4] CDF collaboration, D. Acosta et al., Phys. Rev. Lett. 93, 072001 (2004); D0 collaboration, V.M. Abazov et al., Phys. Rev. Lett. 93, 162002 (2004); BaBAr collaboration, B. Aubert et al., Phys. Rev. D 71, 071103 (2005).

[5] BELLE collaboration, S.-K. Choic et al., Phys. Rev. Lett. 100, 142001 (2008).

[6] LHCb collaboration, A.A. Alves Jr., JINST 3, S08005 (2008).

[7] R. Aaij et al., JINST 8, P04022 (2013).

[8] M. Jacob and G.C. Wick, Ann. Phys. 7, 404 (1959).

[9] LHCb collaboration, R. Aaij et al., Eur.Phys. J.C. 73, 2631 (2013).

[10] LHCb collaboration, R. Aaij et al., Eur.Phys. J.C. 74, 2872 (2014).

[11] M. Butenschoen and B.A. Kniehl, Phys.Rev.Lett. 108, 172002 (2012).

[12] K.-T. Chao et al., Phys. Rev. Lett. 110, 042002 (2013). 
[13] ALICE and LHCb collaborations, Reference pp cross-sections for $\mathrm{J} / \psi$ studies in proton-lead collisions at $\sqrt{s_{N N}}=5.0 \mathrm{TeV}$ and comparisons between ALICE and LHCb results, LHCb-CONF2013-013, ALICE-PUBLIC-2013-002.

[14] LHCb collaboration, R. At et al., JHEP 02, 072 (2014).

[15] E.G. Ferreiro et al., Phys.Rev. C. 88, 047901 (2013); J. Albacete et al., Int. J. Mod. Phys. E 22, 1330007 (2013); F. Arleo and S. Peigné, JHEP 03 , 122, (2013).

[16] Z.C. del Valle et al., Nucl. Phys. A. 926, 236, (2014).

[17] CDF collaboration, A. Abulencis et al., Phys. Rev. Lett. 96, 102002, (2006); CDF collaboration, A. Abulencia et al., Phys.Rev.Lett. 98, 132002 (2007).

[18] LHCb collaboration, R. Aaij et al., Eur. Phys. J. C. 72, 1972 (2012).

[19] LHCb collaboration, R. Aaij et al., Phys. Rev. Lett. 110, 222001 (2013).

[20] LHCb collaboration, R. Aaij et al., Nucl. Phys. B 886, 665 (2014).

[21] LHCb collaboration, R Aaij et al., Phys. Rev. Lett. 112, 222002 (2014).

[22] Belle collaboraton, K. Chilikin et al., Phys. Rev. D 88, 074026 (2013).

[23] BaBar collaboration, B. Aubert et al., Phys. Rev. D 79, 112001 (2009); BaBar collaboration, J.P. Lees et al., Phys. Rev. D 85, 052003 (2012).

[24] F. James, Statistical Methods In Experimental Physics (World Scientific, Singapore, 2006) 270286. 
